\title{
Protetor Esportivo para Pacientes Ortodônticos: Importância e Técnica de Manufatura
}

\section{Sport Mouthguard for Orthodontics Patients: the Importance and a Custom-Made Technique}

\author{
Luciana Sayuri Shida Scarsia ; Ivan Onone Gialain*a
}

\begin{abstract}
aUniversidade de Cuiabá, Programa de Pós-Graduação Stricto Sensu em Ciências Odontológicas Integradas. MT, Brasil. *E-mail: ivan.gialain@kroton.com.br
\end{abstract}

\begin{abstract}
Resumo
O trauma orofacial esportivo em pacientes ortodônticos fixos poderá implicar em lesões com diferentes graus de severidade, reversíveis e irreversíveis, podendo acometer tanto tecidos bucais moles quanto duros. As lesões, normalmente, ocorrem pelo contato destes tecidos com o agente traumatizante e pelo próprio contato do aparelho com os tecidos intrabucais. Portanto, em pacientes sob tratamento ortodôntico com aparelho fixo, a recomendação do uso do protetor bucal deve ser enfatizada, pois, em casos extremos, pode ocorrer também a perda parcial ou total de elementos dentais. O protetor bucal pode ser definido como um dispositivo recomendado para a proteção das estruturas orais de pacientes durante a prática de atividade esportiva. Dependendo da atividade esportiva, como nos esportes de contato, há obrigatoriedade estabelecida por federações e confederações para o seu uso como equipamento de proteção individual. O presente trabalho apresenta o passo a passo de uma técnica simples e de baixo custo para a confecção de um protetor bucal personalizado, através da modificação da técnica convencional, promovendo um alívio durante a moldagem, o que permite o uso confortável e seguro em pacientes ortodônticos. Além disso, o presente artigo reforça a importância do dentista em prescrever e conscientizar, tanto o atleta profissional quanto o amador, no uso deste dispositivo preventivo, que pode ser confeccionado em uma sessão, evitando possíveis prejuízos bucais e resguardando o paciente de danos físicos, psicológicos, sociais e financeiros.
\end{abstract}

Palavras-chave: Protetor Bucal. Esporte. Ortodontia.

\begin{abstract}
Sports orofacial trauma in fixed orthodontic patients may result in different degrees of severity, reversible and irreversible injuries, in soft and hard oral tissues, by their contact with the traumatizing agent and by the device contact with intraoral tissues. Therefore, in these conditions, the recommendation for the use of mouthguards should be emphasized because, in extreme cases, partial or total loss of dental elements may also occur. The mouthguard can be described as a device with the purpose to avoid injuries to patients'oral structures during the practice of sports activity. Depending on the sporting activity, as in contact sports, the use as personal protective equipment is imposed by federations and confederations. The objective of this article is to present the step by step of a simple and low-cost technique for the confection of a personalized mouth guard, through the conventional technique modification, creating a relief during molding, which allows the comfortable and safe use in orthodontic patients. In addition, this article aims to reinforce the dentist's importance in prescribing and raising awareness, both professional and amateur athletes, in the use of this preventive device that can be made in one session, avoiding possible oral damage and also protecting the patient from psychological, social and financial injuries.
\end{abstract}

Keywords: Mouthguard. Sport. Orthodontics.

\section{Introdução}

O protetor bucal pode ser definido como um dispositivo para proteção das estruturas orais quando submetidas a traumas mecânicos resultantes da atividade esportiva. Os primeiros relatos de equipamentos de proteção oral existentes na literatura datam do início do século XX. Nesses relatos há menção sobre um cirurgião-dentista londrino que desenvolveu, em 1890, um dispositivo em guta percha para proteção de um boxeador cobrindo os incisivos. No entanto, mesmo fora do ambiente científico, há relatos de equipamentos semelhantes que são utilizados desde a antiguidade, normalmente, confeccionados com couro, cortiça ou outros materiais macios (DIAS; COTO, 2014; KNAPIK et al., 2007).

É possível encontrar protetores bucais em lojas de artigos esportivos, que são mais comumente utilizados por atletas amadores. Tais protetores são classificados como protetores do tipo I (ou de estoque), que possuem um tamanho predeterminado e, normalmente, não oferecem nenhum tipo de ajuste ou adaptação; ou do tipo II (ou termoformável), em que o dispositivo tem um tamanho predeterminado, mas pode ser levado a um banho de água quente e ser adaptado no formato da arcada do paciente. Ambos tipos de protetores bucais têm pontos negativos e não são os mais recomendados para atividades esportivas. O tipo III (ou individualizado) é confeccionado sobre modelo do paciente e, portanto, oferece melhor adaptação e relação entre o dispositivo protetor e as estruturas orais. O principal material utilizado para confecção dos protetores é o EVA (copolímero de etileno e acetato de vinila) sendo formado com o auxílio de uma plastificadora por pressão positiva ou negativa (ASTM, 2016; PADILHA; 
NAMBA, 2014).

Com o desenvolvimento de regras, para diferentes modalidades esportivas, visando o bem-estar e manutenção da saúde dos atletas, muitas federações e confederações passaram a reconhecer os protetores bucais como equipamento de proteção e, em alguns casos, tornaram o seu uso mandatório em atividades competitivas. Diversos grupos de pesquisa têm realizado levantamentos epidemiológicos acerca de lesões orofaciais, que acometem atletas de diferentes modalidades, assim subsidiando as decisões e recomendações para o uso de equipamento de proteção, de acordo com a necessidade que cada modalidade requer. Como exemplos de obrigatoriedade do uso de protetores bucais se pode citar: artes marciais, como o boxe e o MMA (mixed martial arts), e outras modalidades em categorias escolares ou universitárias (ALLISON; TAMIMI, 2020; KNAPIK et al., 2007).

Dependendo da atividade esportiva há recomendação para que todos os praticantes utilizem protetores bucais, e em condições orais específicas, mesmo que em atividades com menor risco de lesões, a recomendação deve ser mais enfatizada. Esse é o caso de pacientes que estão sob tratamento ortodôntico com aparelhos fixos, já que neste caso o próprio aparelho pode ser um potencial agressor e causar lesões. Por conta dessa condição especial e pela característica estrutural e de volume, que o próprio aparelho ortodôntico promove na adaptação reduzida do protetor bucal, o presente artigo visa demonstrar a importância de seu uso e descrever uma técnica para a confecção de um protetor bucal modificado para o paciente, que utiliza aparelho ortodôntico fixo (COTO et al., 2014; GIALAIN; COTO; DIAS, 2014; PACHECO et al., 2010; SALAM; CALDWELL, 2008).

\section{Desenvolvimento}

\subsection{Principais lesões traumáticas em atividades esportivas}

Diversas atividades esportivas podem expor os atletas a situações de risco para lesões traumáticas na região orofacial. De acordo com a posição anatômica e a prevalência das lesões se estabeleceu que os protetores bucais são mais indicados para a arcada superior. Porém, em casos específicos, podem ser confeccionados também no arco inferior (PADILHA; NAMBA, 2014).

As lesões podem ser divididas em lesões de tecidos moles, lesões em estruturas dentais e lesões em estruturas periodontais. As lesões em tecidos moles são as mais prevalentes e compreendem as abrasões, as contusões e as lacerações, principalmente, na região de lábios, bochechas e língua (SMITH; KRACHER, 1998).

Em relação às lesões em estruturas dentais, os elementos mais comumente acometidos são os anteriores superiores, sendo que o gênero masculino apresenta maior prevalência deste tipo de trauma. Este fato está, possivelmente, relacionado a maior participação desta população em atividades esportivas, que envolvem contato físico (JONES, 2020). As lesões dentais podem ser trincas, fraturas coronárias, fraturas radiculares ou fraturas coronorradiculares, com ou sem a exposição da polpa dental. Além de lesões parciais, em esmalte, dentina e polpa, outras lesões como: concussão, subluxação, luxação lateral, luxação intrusiva, luxação extrusiva e avulsão, podem causar o deslocamento do elemento dentário e, consequentemente, a sua perda (ANDREASEN et al., 2011; ANDREASEN et al., 2012).

Dependendo do local e da severidade das lesões, o tratamento pode variar desde acompanhamento até procedimentos progressivamente mais invasivos, como: restaurações, tratamento endodôntico e até exodontias. A principal estratégia para prevenção das lesões supracitadas, nas práticas esportivas, é o uso de protetores bucais, de preferência do tipo III, individualizado e confeccionado por um cirurgião-dentista (DIAS; COTO, 2014; PADILHA; NAMBA, 2014).

\subsection{Características especiais do paciente em tratamento ortodôntico}

Quando o paciente-atleta possui qualquer dispositivo intraoral, tal objeto pode ser considerado um possível causador ou potencializador de lesões. Entre esses dispositivos podem ser citados: piercings, próteses parciais ou totais removíveis ou mesmo aparelhos ortodônticos, fixos ou removíveis. Sempre que possível é preferencial que o atleta remova todos os objetos e dispositivos durante a atividade física (GIALAIN et al., 2014; SMITH; KRACHER, 1998). Além dos riscos às estruturas orais, danos podem ocorrer aos componentes utilizados na movimentação ortodôntica, atrapalhando o andamento do tratamento.

Salam, em 2008, afirmou que muitos pacientes, principalmente, adolescentes em tratamento ortodôntico, participam de esportes de contato sem o uso de protetores bucais. Nesse período da vida é relatada uma alta incidência de trauma dentário durante a prática desta categoria de esporte em que o contato físico é altamente competitivo. Quando uma criança, em tratamento ortodôntico, sofre algum tipo de injúria orofacial é mais fácil ocorrer um dano adicional aos tecidos moles. Em um estudo no Reino Unido, foi observado que a maior incidência de acidentes esportivos em crianças ocorreu, principalmente, em meninos (RODD; CHESHAM, 1997).

Em um estudo utilizando elementos finitos, foi observado que a presença de protetores bucais é eficaz na diminuição de tensões geradas nas estruturas dentais por impacto frontal. No mesmo estudo foi realizada a comparação entre utilização de brackets metálicos ou cerâmicos na distribuição de forças, e foi concluído que brackets metálicos resultaram em menores níveis de tensão nas simulações realizadas (ALVES et al., 2020).

Apesar da clara necessidade em se recomendar o uso de protetores bucais, um estudo realizado no Reino Unido 
trouxe alguns dados preocupantes acerca do conhecimento da indicação e uso de protetores bucais entre ortodontistas. Segundo o estudo, apenas $61 \%$ dos participantes da pesquisa perguntam a seus pacientes, em tratamento ortodôntico, se praticam algum tipo de esporte de contato, e apenas 55\% dos profissionais confeccionam protetores bucais para os seus pacientes. Os profissionais que não os confeccionam, normalmente, sugerem para seus pacientes adquirirem os protetores em lojas de material esportivo (BUSSELL; BARRETO, 2014).

Como alternativas para diminuir a severidade de incidência de lesões traumáticas, são necessários mais estudos avaliando e comparando os riscos da ortodontia fixa convencional em relação ao uso de ortodontia lingual ou de alinhadores removíveis.

\subsection{Confecção do protetor bucal para o paciente ortodôntico}

A técnica apresentada a seguir é baseada em trabalhos prévios realizados por diferentes autores (COTO et al., 2014; PACHECO et al., 2010). Segundo outros estudos, se têm algumas considerações sobre dimensões e extensões do protetor bucal individualizado. A espessura indicada deve variar entre $2 \mathrm{~mm}$ a $4 \mathrm{~mm}$ na região vestibular, a extensão deve cobrir todos os elementos dentários até a distal do segundo molar. Como normalmente são descritas técnicas de protetores bucais para o arco superior se irá descrever a técnica, utilizando imagens da confecção para o arco inferior. Apesar de normalmente o protetor bucal ser indicado apenas para o arco superior, quando há presença de elementos nos dentes inferiores, que podem aumentar o risco de trauma, é indicado o protetor inferior para proteger os tecidos orais de, por exemplo, o próprio aparelho ortodôntico fixo (DIAS; COTO, 2014).

A confecção segue as seguintes etapas:

1. As arcadas superior e inferior devem ser moldadas com o material de escolha do profissional. Na visão dos autores, como o protetor bucal não necessita de uma adaptação tão precisa como peças protéticas, é aceito inclusive a utilização de alginato;

2. Os modelos de gesso devem ser confeccionados com material com dureza compatível para serem utilizados como modelo de trabalho; após a cristalização e remoção do molde, o modelo deve ser recortado para permitir uma correta conformação do EVA durante a termoplastificação, como visto na Figura 1.

Figura 1 - Modelos da arcada inferior de paciente utilizando aparelho ortodôntico fixo. A - Vista lingual; B - Vista vestibular

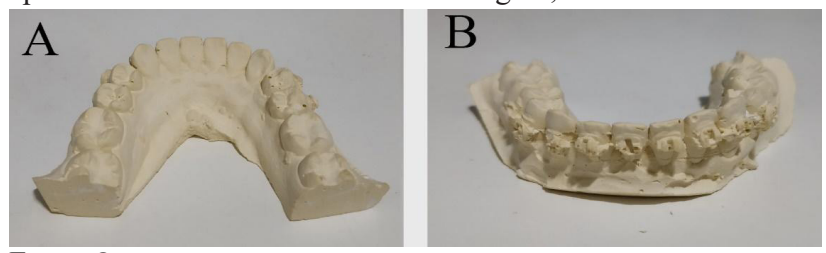

Fonte: Os autores.

3. Com o planejamento ortodôntico em mente, alívios devem ser feitos no modelo de gesso, a fim de aumentar a vida útil do protetor bucal. Lembrando que a movimentação dentária pode causar falta de adaptação do protetor bucal, sendo então necessária a confecção de um novo dispositivo;

4. Outro alívio deve ser confeccionado, a fim de cobrir a região dos bracketes e do fio ortodôntico, para possibilitar a adaptação do protetor bucal. Para o alívio, os autores utilizaram silicone de condensação por ser um material relativamente simples de ser manipulado e ser termofixo (Figura 2). O uso de cera (utilidade ou 7) não é recomendado por se tratar de um material termoplástico, que pode sofrer alterações durante a termoconformação da lâmina de EVA.

Figura 2 - Alívio na região do aparelho feito com silicona de condensação, em amarelo. A. Vista oclusal; B. Vista vestibular

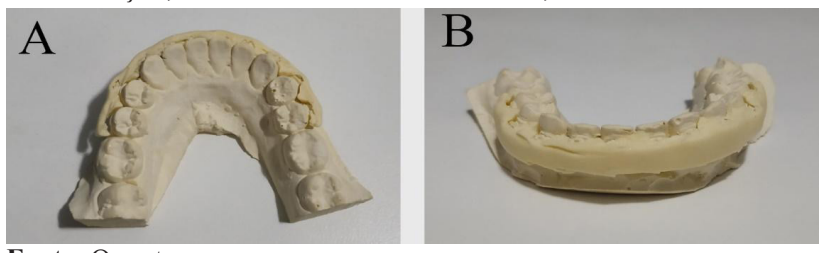

Fonte: Os autores.

5. O modelo com os alívios deve receber uma camada de isolante para gesso.

6. Utilizando uma plastificadora à vácuo se deve confeccionar uma primeira camada utilizando uma placa de EVA (sempre optar pelo tipo soft) com 1 milímetro de espessura. Essa primeira camada deve ser recortada, a fim de recobrir a coroa dos dentes que sofrerão movimentação e servirá como mais um alívio. Tal alívio terá a função de aumentar a vida útil do protetor bucal (Figura 3). Não existe consenso na literatura sobre a vida útil de um protetor bucal, mas é discutido que a manutenção da espessura e adequada adaptação são fatores a serem considerados para indicar a troca do dispositivo (DEL ROSSI; LISMAN; LEYTE-VIDAL, 2007)case-series study. Setting: High school. Patients or Other Participants: Fifteen high school varsity football players aged 15 to 17 years began the investigation; 4 participants lost their mouthguards before the end of the season. Main Outcomes Measure(s.

Figura 3 - Confecção da primeira camada de EVA (camada de alívio). A - Vista lingual; B - Vista vestibular
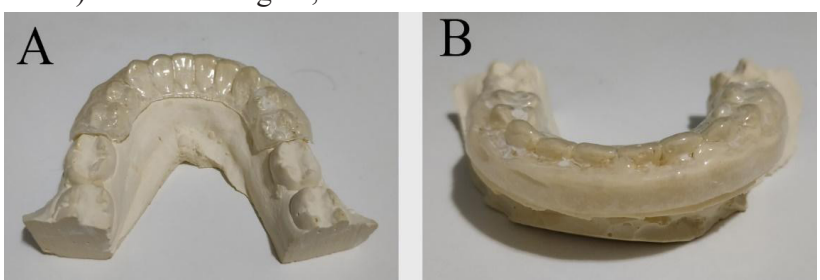

Fonte: Os autores.

7. Sobre a primeira camada deve ser aplicada uma fina camada de vaselina, para impedir a adesão à segunda camada, que formará o protetor bucal.

8. O modelo com o alívio em silicone e em EVA deve ser levado novamente à plastificadora para conformação do protetor bucal, que pode ser realizado com lâminas de EVA de 3 ou 4 milímetros, sendo com uma ou múltiplas lâminas. Alguns autores recomendam utilização de até 4 milímetros de espessura por vestibular, quando confeccionar protetor na arcada superior (GIALAIN et al., 2016; WESTERMAN; STRINGFELLOW; ECCLESTON, 2002). Já na arcada inferior, o protetor terá mais função de proteger os tecidos moles contra lesões causadas pelo próprio aparelho, então, 
uma lâmina de apenas 2 milímetros foi utilizada (Figura 4A).

Figura 4A - Segunda camada de EVA (camada do protetor bucal); 4B - Primeira camada destacada da segunda
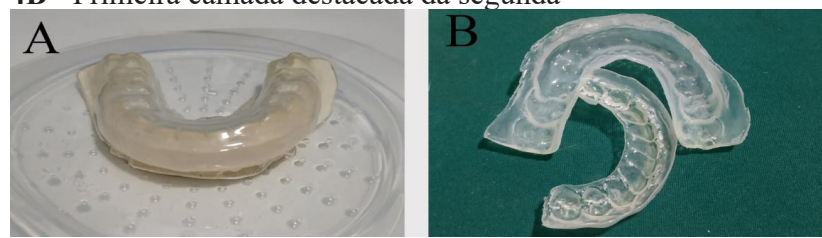

Fonte: Os autores.

9. O protetor pode ser recortado com a utilização de tesoura ou disco de corte e motor de baixa rotação. Para o acabamento e polimento é possível utilizar broca do tipo maxicut, pontas de borracha com abrasivos e discos de feltro ou outros instrumentos utilizados para acabamento e polimento de próteses acrílicas. $\mathrm{Na}$ fase de acabamento e polimento, a primeira camada (de alívio) deve ser removida (Figura 4B).

10. A instalação do protetor bucal também é uma atribuição profissional, pois no ato da instalação pode ser necessária a realização de ajustes para uma correta inserção e remoção do protetor sem machucar nenhuma região. Além disso, é importante realizar ajuste oclusal, quanto maior for o número de contatos entre a porção oclusal do protetor e o arco antagonista, melhor será a distribuição das forças de impacto.

Após a instalação é importante destacar a importância do uso do protetor bucal, em todas as atividades, que envolvam risco de lesão, inclusive nas sessões de treinamento. O protetor deve sempre ser guardado em uma caixa específica para esse fim (utilizar caixas para acondicionamento de aparelhos removíveis), e o dispositivo deve ser lavado antes e depois do uso e mantido sempre em local seco e longe de fontes de calor.

\section{Conclusão}

O presente estudo demonstrou, a partir de uma revisão de literatura, que o paciente-atleta que está sob tratamento ortodôntico com aparelho fixo tem um risco maior de sofrer lesões traumáticas na região orofacial frente aos impactos esportivos. Portanto, independentemente da idade ou nível competitivo, é recomendado o uso de protetores bucais, principalmente, em atividades esportivas com contato. Para possibilitar aos cirurgiões-dentistas confeccionarem os protetores bucais, foi demonstrada uma técnica utilizando materiais e equipamentos comumente encontrados na prática clínica. O principal resultado esperado é que possam ser minimizados os riscos de lesões traumáticas durante atividades esportivas.

\section{Referências}

ALLISON, P.; TAMIMI, F. Mouthguards should be worn in contact sports. British J. Sports Med., v.54, n.17, p.1016-1017, 2020. doi:-10.1136/bjsports-2020-102041.

ALVES, J. C. DE C. et al. Effect of orthodontic bracket type and mouthguard presence on the stress and strain during a frontal impact. Braz. Dent. J., v.31, n.5, p.540-547, 2020. doi:10.1590/0103-6440202002818.
ASTM - American Standards of Testing Of Materials. F697-16 Standard Practice for Care and use of Mouthguards. Annual Book of ASTM Standards, 2016.

ANDREASEN, J.O. et al. Traumatic dental injuries: a manual. New Youk: John Wiley \& Sons, 2011.

ANDREASEN, J.O. et al. Dental Trauma Guide: a source of evidence-based treatment guidelines for dental trauma. Dent. Traumatol., v.28, n.5, p.345-350, 2012. doi: 10.1111/j.16009657.2011.01059.

BUSSELL, M.A.; BARRETO, L.S. The recommendation and provision of mouthguards: A survey of consultant orthodontists in the UK. J. Orthod., v.41, n.2, p.141-146, 2014. doi:10.1179/14 65313313Y.0000000082.

COTO, N.P. et al. Protetor bucal individualizado, para esporte, específico para Ortodontia. Rev. Assoc. Paul. Cir. Dent., v.68, n.2, p.96-99, 2014.

DEL ROSSI, G.; LISMAN, P.; LEYTE-VIDAL, M. A. A preliminary report of structural changes to mouthguards during 1 season of high school football. J. Athletic Training, v.42, n.1, p.47-50, 2007.

DIAS, R.B.; COTO, N.P. Odontologia do esporte: uma abordagem multiprofissional. Rio de Janeiro: Medbook, 2014.

GIALAIN, I.O. et al. A three-dimensional finite element analysis of the sports mouthguard. Dent. Traumatol., v.32, n.5, p.409-415, 2016. doi: 10.1111/edt.12265.

GIALAIN, I. O. et al. Mouthguard: a new technique for the partially edentulous patient. Dent. Traumatol., v.30, n.5, p.411414, 2014. doi:10.1111/edt.12106.

GIALAIN, I.O.; COTO, N.P.; DIAS, R.B. University student's dental and maxillofacial fractures characteristics and epidemiology in sports. Acta Scie. Health Sci., v.36, n.2, p.293, 2014. doi: 10.4025/actascihealthsci.v36i2.19221.

JONES, L.C. Dental trauma. Oral Maxillofacial Surg. Clin. North Am., v.32, n.4, p.631-638, 2020. doi:10.1016/j.coms.2020.07.009.

KNAPIK, J.J. et al. Mouthguards in Sport Activities. Sports Med., v.37, n.2, p.117-144, 2007. doi:10.2165/00007256-20073702000003 .

PACHECO, G. et al. The orthodontic sports protection appliance. JCO, v.44, n.1, p.41-44, 2010.

PADILHA, C.; NAMBA, E. L. Protetores bucais esportivos - Tudo o que o cirurgião-dentista precisa saberBalneário Camboriu: 893 Editora, 2014.

RODD, H.D.; CHESHAM, D.J. Sports-related oral injury and mouthguard use among Sheffield school children. Comm. Dent. Health, v.14, n.1, p.25-30, 1997.

SALAM, S.; CALDWELL, S. Mouthguards and orthodontic patients. J. Orthod., v.35, n.4, p.270-275, 2008. doi: $10.1179 / 14653120722779$.

SMITH, W. S.; KRACHER, C. M. Sports-related dental injuries and sports dentistry. Dental assistant (Chicago, Ill.: 1994), v. 67, n.3, p.12-16, 1998.

WESTERMAN, B.; STRINGFELLOW, P. M.; ECCLESTON, J. A. EVA mouthguards: How thick should they be? Dent. Traumatol., v.18, n.1, p.24-27, 2002. doi: 10.1034/j.16009657.2002.180103. 